
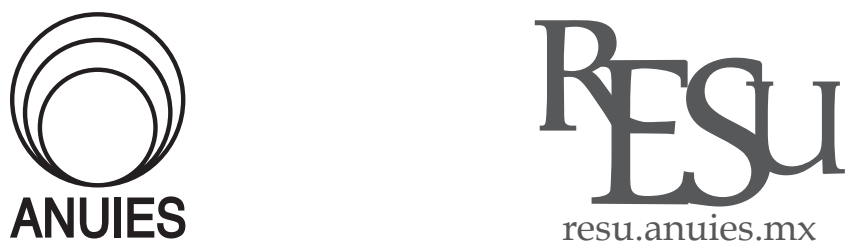

ARTí́CULO

\title{
Deserción Estudiantil en la Educación Superior Técnico- Profesional: Explorando los factores que inciden en alumnos de primer año
}

\author{
Student Dropout in Technical-Professional Higher Education: Exploring \\ factors that influence freshmen students
}

\author{
Felipe Ignacio González Catalán* y Karin Jeanette Arismendi Vera** \\ * Profesor Magíster en Educación mención Evaluación de Aprendizajes por la Pontificia Universidad \\ Católica de Valparaíso. \\ Correo electrónico: felipe.gonzalez.c@pucv.cl \\ ** Profesora de Castellano y Comunicación de la Pontificia Universidad Católica de Valparaíso y \\ Magíster en Educación mención Currículum Escolar por la Pontificia Universidad Católica de Chile. \\ Correo electrónico: karismendi@duoc.cl
}

Recibido el 29 de noviembre de 2017; aceptado el 25 de septiembre de 2018.

\begin{abstract}
Resumen
Los estudios en torno a la deserción a nivel universitario ha sido foco de interés para los expertos en el ámbito educativo, sobre todo a raíz que la tasa de abandono en la última década ha oscilada entre el 30\% y el 50\% (Centro de Microdatos, 2008). Esto, ha motivado el desarrollo de la investigación en el área en distintos países de américa latina. En Chile, el estudio de la deserción universitaria ha estado asociado a modelos conceptuales (Himmel, 2002), evaluación de costos económicos (González \& Uribe, 2005) y la generación modelos predictivos que reconocen los factores que explicarían el fenómeno (Villa \& Barriga, 2010; Mizala, 2011). Estas indagaciones han sido en el contexto de la universidad, mientras que en la educación técnico-profesional el foco ha estado en describir la situación más que la generación de modelos predictivos. Dado esto, mediante este trabajo se busca determinar las variables que explican significativamente la deserción de los estudiantes en una institución técnico-profesional en la cohorte 2014-2016, a través de un estudio con alcance explicativo y no experimental. La muestra correspondió a 1876 estudiantes de primer año de un instituto técnico-profesional de la región de Valparaíso y los datos fueron procesados con el software R,
\end{abstract}


aplicando un modelo lineal generalizado con distribución de errores binomial y función de vínculo Logit. Los resultados muestran que los factores con mayor significancia estadística corresponden al género, año de egreso de enseñanza media y jornada de estudio. Asimismo, es importante precisar que esto dependerá de la escuela de formación, pues el modelo varía de acuerdo con las particularidades de cada campo de formación.

Palabras clave: Deserción universitaria; Modelos de deserción; Modelo causal; Variables predictivas; Educación técnico-profesional.

\begin{abstract}
The studies about the desertion at university level has been a focus of interest for experts in the educational field, especially since the dropout rate in the last decade has oscillated between 30\% and 50\% (Centro de Microdatos, 2008). This has motivated the development of research in the area in different countries of Latin America. In Chile, the study of college dropout has been associated with conceptual models (Himmel, 2002), evaluation of economic costs (González \& Uribe, 2005) and the generation of predictive models that allow us to recognize the factors that would explain to this phenomenon (Villa \& Barriga, 2010; Mizala, 2011). These inquiries have been in the context of the university, while in technical-professional education the focus has been on describing the situation rather than generation of predictive models. Given this, this work aims to determine the variables that significantly explain the dropout of students in a technical-professional institution in the 2014-2016 cohort, through a study with explanatory scope and non-experimental. The sample corresponded to 1876 first-year students and the data were processed with the R software, applying a generalized linear model with binomial error distribution and Logit link function. The results show that the factors with greater statistical significance correspond to the gender, year of graduation of secondary education and school hours. Likewise, it is important to specify that this will depend on the training school, since the model varies according to the particularities of each training field.
\end{abstract}

Keywords: College dropouts; Dropouts models; Causal model; Predictor variables; Technical education. 


\section{Introducción}

—1 interés por el tema de la deserción universitaria en los últimos años -1 se ha profundizado, es así como, desde los años setenta, ha sido foco de preocupación en países como Estados Unidos y Canadá; al igual que en otras naciones miembros de la OECD, las tasas de estudiantes que abandonan la educación superior han alcanzado cifras históricas, pues estos datos oscilan entre el 30\% y 50\%, siendo aún más alto en países sin selección para ingresar a la formación profesional (Centro de Microdatos, 2008). En relación a este tipo de estudios, y particularmente en el ámbito nacional, en la última década los trabajos se han enfocado principalmente en la discusión de modelos conceptuales (Himmel, 2002), así como también de los costos asociados que tiene la deserción en este nivel (González y Uribe, 2005). Por lo mismo, en la actualidad se ha hecho necesario profundizar la investigación en este ámbito, orientándola hacia la comprensión de este fenómeno a partir de modelos empíricos, produciéndose este cambio de foco en gran parte de los países en Latinoamérica.

Es así como han sido desarrollados estudios en distintas naciones de Latinoamérica. En Colombia, la deserción universitaria se ha considerado un problema estructural de la educación superior, por lo mismo, Malagón, Soto y Eslava (2007) han estudiado este fenómeno entre los periodos 1998 a 2004 en la Universidad de Los Llanos, determinando que las causas son de tipo académicas y no socioeconómicas. Contrario a esto, Ariza y Marín (2009), en su trabajo en la carrera de Psicología, han concluido que el factor socioeconómico tiene un alto nivel explicativo en lo referido al fenómeno de la deserción. En este mismo contexto, Medellín (2010) destaca que los alumnos universitarios que manifiestan una mayor autonomía tienen menor posibilidad de desertar. En tanto, Olave, Rojas y Cisneros (2013) señalan que los motivos de la deserción están en relación con la alfabetización académica.

En Cuba, López, Marín y García (2012) han caracterizado la deserción en el primer año de un programa de formación de médicos en el período 20072009. A partir de ello, concluyeron que este fenómeno es de carácter multicausal, siendo los factores más relevantes las dificultades de aprendizaje y la falta de motivación.

En México, Huesca y Castaño (2007) señalan que la deserción de la educación universitaria se debe a un síndrome que han denominado como atraso del sistema escolar. A esto, se suma la investigación de Rodríguez y Hernán- 
dez (2008), los que declaran que la deserción no puede ser explicada desde el punto de vista económico, familiar, integración o desempeño académico, sino que por núcleos explicativos graduales.

En Venezuela, Colmenares y Delgado (2008) exponen que el abandono universitario es producto del bajo rendimiento de los estudiantes y esto está fuertemente asociado a las motivaciones de logro de los alumnos. En el caso de Perú, Mori (2014) concluye que dentro de las variables que explican la deserción, la primera apunta al elemento institucional, seguida de los intereses vocacionales, los académicos y, por último, los económicos.

En Chile, los estudios se han enfocado a un trabajo tanto conceptual como empírico. Uno de estos es lo realizado por Díaz (2008), quien busca explicar la deserción como resultado de la motivación positiva o negativa, la que se ve afectada tanto por la integración académica como social del estudiante a la institución educativa. Para darle continuidad a esto, Villa y Barriga (2010) proponen un modelo para predecir la deserción, reconociendo que las variables que mejor explican esto son los ingresos familiares y el rendimiento académico. Otro de los aportes es el trabajo de Mizala, Hernández y Makovech (2011), quienes se interesaron en abordar las determinantes de la elección y deserción en la carrera de pedagogía, concluyendo que los aspectos de mayor influencia son: presión familiar, la necesidad del estudiante de trabajar y aportar económicamente a su núcleo, el embarazo y paternidad juvenil y el índice de reprobación. En esta misma línea, Celis, Flores, Reyes y Venegas (2013) señalan que la reprobación de asignaturas al primer año es un aspecto altamente relevante para decidir la permanencia en la educación superior.

Finalmente, los datos del Sistema de Información de Educación Superior (SIES) (MINEDUC, 2012) indican que "al segundo año, un 38\% de los estudiantes universitarios, un 55\% de los estudiantes de IP y un 53\% de los estudiantes de CFT ya no se encuentra matriculado en la misma institución a la que ingresaron" (MINEDUC, 2012: 2). Esto constituye un campo de investigación prometedor y necesario de ser explorado dada la complejidad del fenómeno y considerando que la mayor parte de los estudios se concentra en las universidades.

\section{Deserción estudiantil en la educación superior}

Distintas son las propuestas para abordar la deserción, una de ellas es la de Gordillo (1970) quien comprende este fenómeno como un proceso donde el 
número de alumnos matriculados inicialmente en un programa de formación no logra seguir la trayectoria normal de su carrera, ya sea por retirarse o por demorar más tiempo en alcanzar el egreso. Esto es compartido por Nieto (1981) quien solo agrega que este proceso está asociado al alejamiento de metas que se habían definido al iniciar la formación (Torres, 2012). En general, quienes han estudiado el tema comparten la conceptualización, entendiendo este como un proceso de abandono prematuro de un programa de estudio, es decir, antes de cumplir con la meta formativa que se han propuesto (Tinto, 1982; Páramo \& Correa, 1999; Himmel, 2002; Castaño, Gallón, Gómez \& Vásquez, 2004; González, 2006; Díaz, 2008; Torres, 2012).

Las distintas conceptualizaciones establecen tipos o clasificaciones en torno a la deserción. Es así como Tinto (1982) señala que la temporalidad permite establecer diferencias, ya que este abandono puede tener un carácter transitorio o permanente. En el primer tipo, el alumno decide revertir la situación de abandono en la medida que no existan factores que afecten en la decisión de retomar el proceso de formación; en el segundo tipo, existe una situación que se torna permanente, existiendo causas concretas que imposibilitan el retomar los estudios (De los Ríos \& Canales, 2007).

Por su parte, Himmel (2002) propone una clasificación asociada a la naturaleza del proceso de abandono, vale decir, voluntario u obligatorio. El abandono voluntario es la renuncia no informada a la institución; en el caso del obligatorio, se produce a raíz de una decisión de la organización educativa, la que se fundamenta en las definiciones consignadas en los reglamentos propios. Dado esto, la deserción no corresponde a una situación aislada y descontextualizada, por tanto, renunciar a una carrera no implica necesariamente el abandono de la institución, por tanto, el desertar estará asociado específicamente a la salida de un estudiante de la educación superior de manera definitiva (Himmel, 2002).

Al igual que Tinto (1982), para Castaño, Gallón, Gómez y Vásquez (2004), existen diferencias entre tipos generales de abandono. El primero, alude a la deserción relacionada con el tiempo, al proceso de ausencia recurrente o permanente del proceso formativo, pese a encontrarse matriculado en una carrera específica. El segundo tipo se vincula con la deserción en relación con el espacio, lo que implica un cambio de programa académico o de casa de estudios, así como también el abandono definitivo del sistema educativo.

Para Torres (2012) se hace posible abordar la deserción desde una perspectiva individual, entendiéndola en relación con los propósitos que tienen los estudiantes al ingresar a la educación superior, significando, para quien 
ejerce el acto de desertar, un fracaso individual en el proceso de completar un determinado curso de acción. La otra perspectiva es desde la institución, pues para una organización educativa implica una serie de intereses, dado que el desertor genera una vacante que podría haber sido ocupada por un alumno que no provocara una pérdida de recursos para la institución.

Finalmente, Páramo y Correa (1999) señalan que la deserción no se trata únicamente del abandono definitivo de la sala de clases, sino que también de la formación académica, tratándose de una decisión personal que responde a la falta de éxito frente a las exigencias del contexto. Desde esta concepción, los autores precisan seis tipos de deserción: a) deserción total, correspondiente al abandono definitivo de la formación académica individual; b) deserción discriminada por causas, la que se asocia a que existen causas que conllevan a optar por el abandono y que permitirá especificar el acto de desertar; c) deserción por facultad, relacionada con el cambio de escuela, unidad académica o facultad producto de que aquel espacio no satisface intereses y/o motivaciones; d) deserción por programa, vinculada con la carrera a raíz de que esta no logró cumplir con la expectativas del individuo o que en su defecto el éxito en ese programa no fue el esperado; e) deserción en el primer semestre de la carrera, asociada a la escasa o nula adaptación al contexto universitario y f) deserción acumulada, la que responde a la sumatoria de los tipos descritos previamente.

\section{Modelos en torno a la Deserción}

Variados son los modelos existentes para el estudio de la deserción, por lo mismo, Barrios (2011) señala que los enfoques para el análisis de la deserción pueden clasificarse en cinco categorías, las que dependerán de la importancia de las variables explicativas (Braxton, Johnson \& Shaw-Sullivan, 1997). Es así como se precisan modelos de corte psicológico, económico, sociológico, organizacional y de interacciones, además de un enfoque integrado que rescata distintos elementos de los otros (St. John, Cabrera, Nora \& Asker, 2000).

Desde la mirada psicológica, el interés está en la conducta del sujeto, pues se asume que la decisión de desertar no viene dada por conductas previas, sino que es el resultado del debilitamiento de las intenciones o expectativas iniciales. Esto, a partir de la propia evaluación del estudiante acerca de la experiencia vivida en el contexto de la educación superior, a fin de terminar si ha sido un éxito o un fracaso (Díaz, 2008). En tanto, el enfoque económico 
corresponde al proceso de análisis y evaluación permanente que realizan los sujetos, a propósito de los beneficios sociales y económicos. A partir de esto, se opta por aquello que cada individuo considera que le está entregando una mayor cantidad de beneficios (Apaza \& Huamán, 2012).

Respecto del modelo sociológico, este pone el acento en que la decisión de desertar de la formación académica se encuentra altamente influenciada por factores externos. En este sentido, si las influencias que recibe el estudiante no se direccionan positivamente, esto impactará en el desempeño académico, la integración social, la satisfacción y compromiso con la institución y formación, llevando, por lo tanto, a que opte por desertar (Díaz, 2008).

Por otra parte, el enfoque organizacional asume que la deserción depende directamente de las cualidades de la organización educativa en el proceso de integración social y específicamente en la decisión de abandono por parte de los alumnos (Berger \& Milem 2000). Desde este, es altamente relevante la calidad docente y la participación de los estudiantes en el contexto de la sala de clases, pues ambos elementos inciden directamente en el proceso de integración (Braxton et al. 1997 citado en Díaz, 2008). A esto también se suman variables como los beneficios proporcionados por la organización a los estudiantes en áreas como salud, deportes, cultura, apoyo académico y docente (Donoso \& Schiefelbein, 2007).

El modelo de interacciones busca explicar la deserción partir de la existencia de una relación ajustada entre el estudiante y lo entregado por la institución educativa, lo que implica una integración a partir de las experiencias académicas y sociales. Esto significa que la permanencia de un estudiante dentro de una organización dependerá de si, en el proceso de interacción con el establecimiento, se logra percibir la existencia de beneficios que superen los costos personales y que, por ende, motiven al alumno a permanecer (Díaz, 2008; Larroucau, 2013). Esto implica que en la medida que el alumno transita en la educación superior son diversas las variables que una institución debiese considerar en el proceso de adaptación, dado que el sujeto que ingresa trae consigo un conjunto de características que influirán en su experiencia en el nivel formativo universitario. Por su parte, el modelo integrado implica la interacción de variados factores, los que ya han sido considerados por los modelos previos.

A propósito de lo revisado, es relevante recalcar que existen dos dimensiones que son trascendentales en el estudio de la permanencia, a saber, la integración académica e integración social (Díaz, 2008). No obstante, es importante precisar que, si bien ambas dimensiones cobran gran importancia 
para predecir la persistencia o deserción en la educación, estas no tienen el mismo nivel de relevancia en todos los estudiantes y, por lo tanto, en todas las carreras e instituciones (Apaza \& Huamán, 2012).

A fin de sintetizar las ideas ya expuestas, se integra en la tabla 1, tomada de García (2014), una serie de variables de la dimensión integración y académica, tanto individuales como organizacionales, que pueden influir en el proceso de deserción de la educación superior.

Tabla 1

Dimensiones y variables asociadas al estudio de la deserción universitaria

\begin{tabular}{|c|c|c|}
\hline \multicolumn{3}{|c|}{ Factores Individuales } \\
\hline Demográficos & Socioeconómicos & Académicos \\
\hline Sexo & Ingreso del hogar & Promedio de enseñanza media \\
\hline Edad & Nivel educativo de los padres & $\begin{array}{l}\text { Administración del establecimiento de } \\
\text { educación media }\end{array}$ \\
\hline Nacionalidad & Ocupación de los padres & $\begin{array}{l}\text { Tipo de establecimiento de educación } \\
\text { media }\end{array}$ \\
\hline Estado civil & Actividad económica & Horas dedicadas al estudio \\
\hline Lugar de residencia & Cantidad de horas de trabajo & Motivación al ingresar a la carrera \\
\hline Cantidad de hijos & Financiamiento de estudios & Rendimiento académico el primer año \\
\hline \multicolumn{3}{|c|}{ Factores Organizacionales } \\
\hline Políticas académicas & Plan de Estudio & Recursos \\
\hline Mecanismo de admisión & Duración del programa & Formación de los docentes \\
\hline Orientación vocacional & Flexibilidad curricular & Relación docente-alumno \\
\hline Comunicación institucional & Variedad de oferta horaria & Servicios de bienestar estudiantil \\
\hline Condición alumno regular & Cantidad de horas de clase & Becas \\
\hline Prácticas de enseñanza & Mecanismos de evaluación & Infraestructura y equipamiento \\
\hline Seguimiento alumnos & $\begin{array}{l}\text { Estrategias innovadoras para primer } \\
\text { año }\end{array}$ & Inversión para la mejora institucional \\
\hline Tutorías & $\begin{array}{l}\text { Dificultad de las asignaturas de } \\
\text { primer año }\end{array}$ & Cultura o clima de la institución \\
\hline
\end{tabular}

Fuente: García, A. (2014). Rendimiento académico y abandono universitario. Modelos, resultados y alcances de la producción académica en la argentina (adaptación). 


\section{Método}

\section{Tipo de Investigación y alcance}

Esta investigación se enmarca en un paradigma positivista, utilizando herramientas de tipo cuantitativa, transversal con alcance explicativo cuyo diseño es de tipo no experimental.

\section{Unidad de análisis, universo y muestra}

El universo de estudio han sido los estudiantes de primer año de formación técnico-profesional en jornada diurna y vespertina de un instituto profesional acreditado con sede en la región de Valparaíso. En términos de organización, este se encuentra distribuido en escuelas: administración y negocios, con carreras del área de marketing, administración de empresas, auditoría, comercio exterior y logística; comunicación, que cuenta con programas formativos en el ámbito audiovisual, actuación, sonido, publicidad y comunicación social; diseño, con planes en las áreas gráficas, vestuario e industrial; informática y telecomunicaciones, con especialidades asociadas a programación, proyectos informáticos, redes y telecomunicaciones; y salud, cuya formación está vinculada con la actividad física.

Esta casa de estudios, en su admisión, no considera un proceso de selección, esto significa que cualquier persona que desee matricularse puede hacerlo, siempre que tenga su licencia o certificado que compruebe haber completado la educación secundaria. Con el propósito de tener un panorama más claro de las competencias de entrada de los alumnos que ingresan es que, año a año, se realizan evaluaciones diagnósticas en las áreas de lenguaje y matemática. A partir de los resultados obtenidos, aquellos estudiantes que no logran un desempeño mínimo del 70\% de logro en cada una de estas instancias deberán cursar asignaturas de nivelación.

La unidad de análisis corresponde al índice de deserción de una muestra correspondiente a los estudiantes pertenecientes a las cohortes comprendidas entre los años 2014 y 2016. Esta selección responde a que la institución que está siendo estudiada comenzó a tener registro de los resultados de las evaluaciones diagnósticas y a estandarizar el proceso para todas sus sedes desde el año 2014. A continuación, es posible visualizar un resumen de la muestra utilizada. 
Tabla 2

Distribución de frecuencias por género en los estudiantes de primer año entre los periodos 2014-2016

\begin{tabular}{ccc}
\hline Género & Frecuencia & Porcentaje \\
\hline Masculino & 1101 & $58,69 \%$ \\
\hline Femenino & 775 & $39,22 \%$ \\
\hline
\end{tabular}

Tabla 3

Distribución de frecuencias por tipo de establecimiento en los estudiantes de primer año entre los periodos 2014-2016

\begin{tabular}{lcc}
\hline \multicolumn{1}{c}{ Tipo de Establecimiento } & Frecuencia & Porcentaje \\
\hline Particular Subvencionado Técnico-Profesional & 249 & $13,27 \%$ \\
\hline Particular Subvencionado Científico-Humanista & 754 & $40,19 \%$ \\
\hline Municipal Científico-Humanista & 173 & $9,22 \%$ \\
\hline Municipal Técnico-Profesional & 619 & $33,00 \%$ \\
\hline Particular Científico-Humanista & 81 & $4,32 \%$ \\
\hline
\end{tabular}

Tabla 4

Distribución de frecuencias por año de ingreso en los estudiantes de primer año entre los periodos 2014-2016

\begin{tabular}{ccc}
\hline Año de Ingreso a la Educación Superior & Frecuencia & Porcentaje \\
\hline 2014 & 753 & $40,14 \%$ \\
\hline 2015 & 672 & $35,82 \%$ \\
\hline 2016 & 451 & $24,04 \%$ \\
\hline
\end{tabular}


Tabla 5

Distribución de frecuencias por escuela en los estudiantes de primer año entre los periodos 2014-2016

\begin{tabular}{lcc}
\hline \multicolumn{1}{c}{ Escuela } & Frecuencia & Porcentaje \\
\hline Administración y Negocios (AyN) & 835 & $44,51 \%$ \\
\hline Comunicación (C) & 124 & $6,61 \%$ \\
\hline Diseño (D) & 209 & $11,14 \%$ \\
\hline Informática y Telecomunicaciones (IyT) & 459 & $24,47 \%$ \\
\hline Salud (S) & 249 & $13,27 \%$ \\
\hline
\end{tabular}

\section{Identificación de variables y definición operacional}

Las variables en estudio son, por una parte, la deserción de la educación superior (variable dependiente: $Y$ ) y, por la otra, el promedio de enseñanza media (variable independiente: $X_{1}$ ), el establecimiento de procedencia $\left(X_{2}\right)$, los resultados de la evaluación diagnóstica de lenguaje $\left(X_{3}\right)$, los resultados de la evaluación diagnóstica de matemática $\left(X_{4}\right)$, el lugar de residencia del alumno $\left(X_{5}\right)$, el género $\left(X_{6}\right)$ y el año de egreso de la enseñanza media $\left(X_{7}\right)$ y la jornada en la que asiste a clases $\left(X_{8}\right)$. En este estudio, se entenderá la deserción estudiantil como la decisión de permanencia (0) o abandono (1) de sus estudios de educación superior, excluyendo a aquellos sujetos que realizan suspensión o solicitan cambio de carrera. Como un primer grupo de variables independientes se presentan aquellas que son de carácter cuantitativo como: el promedio de notas de enseñanza media, entendida como la calificación reportada por el estudiante a través de su concentración de notas al momento de matricularse en una carrera específica (variable de intervalo); los resultados de las evaluaciones diagnósticas de lenguaje y matemática, correspondientes al porcentaje de logro alcanzado en cada una de las instancias evaluativas de manera independiente (variable de razón) y el año de egreso de la enseñanza media (variable de intervalo). El segundo grupo de 
variables, corresponden a aquellas de carácter nominal y que en las tablas 6, 7,8 y 9 se precisa su codificación.

Figura 1

Variable dependiente e independiente

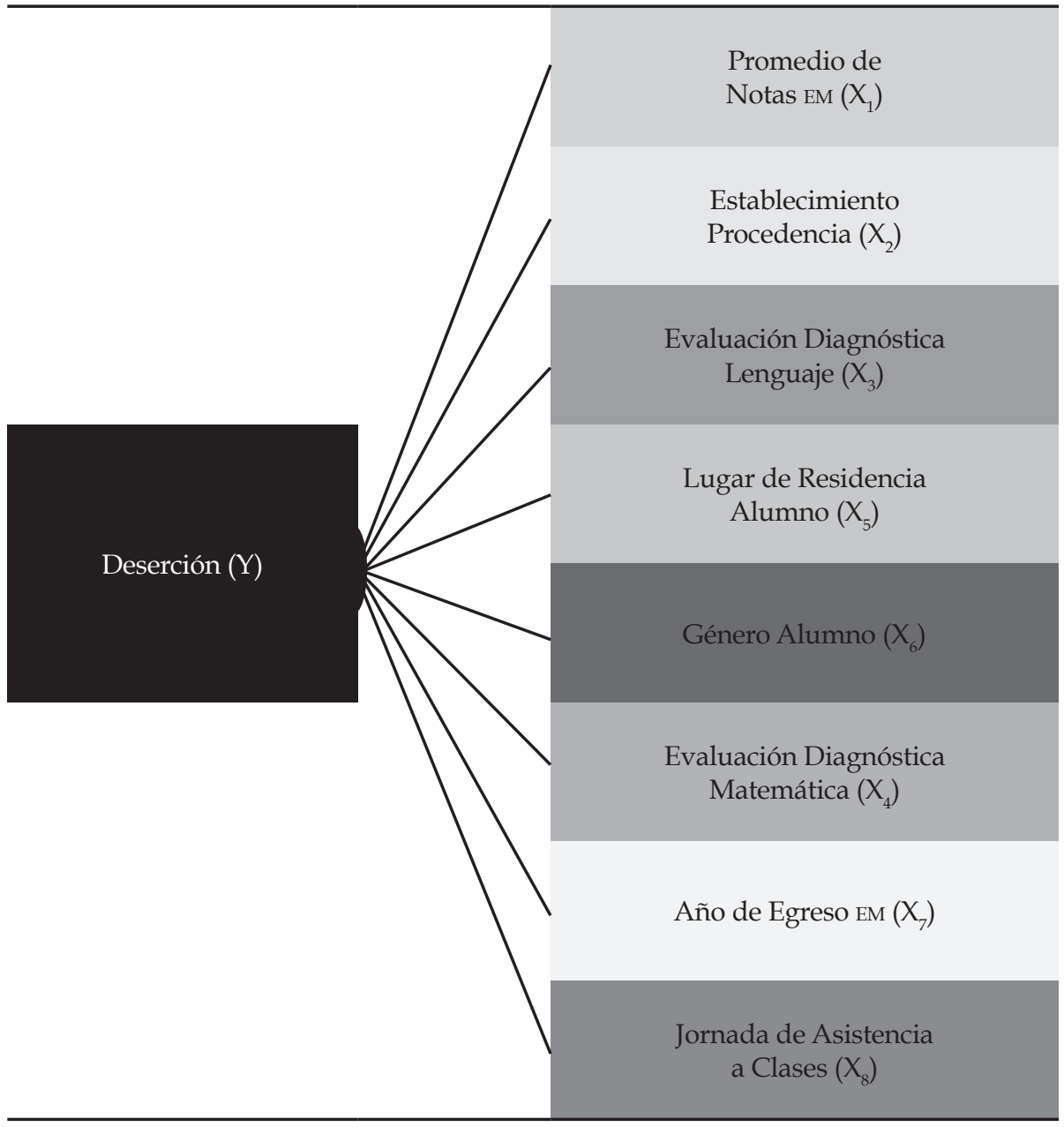


Tabla 6

Variables nominales codificadas asociadas al tipo de establecimiento

\begin{tabular}{lc}
\multicolumn{1}{c}{ Tipo de Establecimiento } & Código \\
\hline Municipal Científico-Humanista & 1 \\
\hline Municipal Técnico-Profesional & 2 \\
\hline Particular Subvencionado Científico-Humanista & 3 \\
\hline Particular Subvencionado Técnico-Profesional & 4 \\
\hline Particular Científico-Humanista & 5 \\
\hline
\end{tabular}

Tabla 7

Variables nominales codificadas asociadas al lugar de procedencia

\begin{tabular}{|c|c|c|c|c|c|}
\hline Comuna & Código & Comuna & Código & Comuna & Código \\
\hline Algarrobo & 1 & Catemu & 14 & El Tabo & 27 \\
\hline Alhue & 2 & Chañaral & 15 & Estación Central & 28 \\
\hline Alto Hospicio & 3 & Chile Chico & 16 & Hijuelas & 29 \\
\hline Antofagasta & 4 & Coinco & 17 & Hualañé & 30 \\
\hline Cabildo & 5 & Con - Con & 18 & Illapel & 31 \\
\hline Cabo de Hornos & 6 & Constitución & 19 & Iquique & 32 \\
\hline Calama & 7 & Copiapó & 20 & Isla de Pascua & 33 \\
\hline Calera & 8 & Coquimbo & 21 & La Calera & 34 \\
\hline Calera de Tango & 9 & Coyhaique & 22 & La Cruz & 35 \\
\hline Calle Larga & 10 & Curacaví & 23 & La Ligua & 36 \\
\hline Cartagena & 11 & Curicó & 24 & La Pintana & 37 \\
\hline Casablanca & 12 & $\begin{array}{l}\text { Diego de } \\
\text { Almagro }\end{array}$ & 25 & La Unión & 38 \\
\hline Castro & 13 & El Quisco & 26 & Limache & 39 \\
\hline
\end{tabular}

Continúa... 
F. I. González C. / K. J. Arismendi V. / Revista de la Educación Superior 47 (188) (2018) 109-137

\begin{tabular}{|c|c|c|c|c|c|}
\hline Comuna & Código & Comuna & Código & Comuna & Código \\
\hline Linares & 40 & Peralillo & 57 & Salamanca & 74 \\
\hline Litueche & 41 & Petorca & 58 & San Antonio & 75 \\
\hline Llay Llay & 42 & Pirque & 59 & San Felipe & 76 \\
\hline Los Andes & 43 & Providencia & 60 & San Fernando & 77 \\
\hline Los Vilos & 44 & Puchuncaví & 61 & San Vicente & 78 \\
\hline Machalí & 45 & Puente Alto & 62 & Santa Cruz & 79 \\
\hline Macul & 46 & Puerto Varas & 63 & Santa María & 80 \\
\hline Maipú & 47 & Putaendo & 64 & Santiago & 81 \\
\hline Maitencillo & 48 & Putre & 65 & Santo Domingo & 82 \\
\hline Nancagua & 49 & Quillota & 66 & Temuco & 83 \\
\hline Navidad & 50 & Quilpué & 67 & Valdivia & 84 \\
\hline Nogales & 51 & Quintero & 68 & Vallenar & 85 \\
\hline Ñuñoa & 52 & Rancagua & 69 & Valparaíso & 86 \\
\hline Olmué & 53 & Rengo & 70 & Villa Alemana & 87 \\
\hline Ovalle & 54 & Requinoa & 71 & Viña del Mar & 88 \\
\hline Papudo & 55 & Rinconada & 72 & Zapallar & 89 \\
\hline Peñablanca & 56 & Romeral & 73 & & \\
\hline
\end{tabular}

Tabla 8

Variables nominales codificadas asociadas a la jornada de asistencia a clases

\begin{tabular}{|c|c|}
\hline Jornada & Código \\
\hline Diurna & 1 \\
\hline Vespertina & 2 \\
\hline
\end{tabular}


Tabla 9

Variables nominales codificadas asociadas al género

\begin{tabular}{lcc}
\hline & Género & Código \\
\hline Femenino & 1 \\
\hline Masculino & 2 \\
\hline
\end{tabular}

\section{Objetivos del Estudio}

\section{Objetivo General}

Determinar las variables que explican significativamente la deserción de los estudiantes de una institución de la educación superior técnico-profesional en la cohorte 2014-2016.

\section{Objetivos Específicos}

- Identificar las variables que explican significativamente la deserción de los estudiantes de la educación superior técnico-profesional en cada una de las escuelas.

- Identificar las dinámicas de organización de la deserción de los estudiantes de la educación superior en cada una de las escuelas.

\section{Preguntas e hipótesis de investigación}

En la tabla 10, se exponen las preguntas que orientan la investigación y que, a su vez, han permitido formular una serie de hipótesis, las que serán retomadas en el apartado de los resultados. 
Tabla 10

Preguntas e hipótesis de investigación

\begin{tabular}{|c|c|}
\hline Preguntas & Hipótesis de trabajo (o investigación) \\
\hline \multirow{8}{*}{$\begin{array}{l}\text { 1. ¿Qué variables explican } \\
\text { significativamente la deserción de } \\
\text { los estudiantes entre los periodos } \\
\text { 2014-2016? }\end{array}$} & $\begin{array}{l}\text { 1.1. El promedio de las notas de enseñanza media afecta de manera } \\
\text { significativa la deserción de los estudiantes. }\end{array}$ \\
\hline & $\begin{array}{l}\text { 1.2. La evaluación diagnóstica de lenguaje afecta significativamente la } \\
\text { deserción de los estudiantes. }\end{array}$ \\
\hline & $\begin{array}{l}\text { 1.3. La evaluación diagnóstica de matemática afecta significativamente } \\
\text { la deserción de los estudiantes. }\end{array}$ \\
\hline & $\begin{array}{l}\text { 1.4. El establecimiento educacional de procedencia afecta de manera } \\
\text { significativa la deserción de los estudiantes. }\end{array}$ \\
\hline & $\begin{array}{l}\text { 1.5. El año de egreso de la enseñanza media afecta de manera } \\
\text { significativa la deserción de los estudiantes. }\end{array}$ \\
\hline & $\begin{array}{l}\text { 1.6. El género del alumno afecta significativamente la deserción de la } \\
\text { educación superior. }\end{array}$ \\
\hline & $\begin{array}{l}\text { 1.7. El lugar de procedencia del alumno afecta de manera significativa la } \\
\text { deserción de la educación superior. }\end{array}$ \\
\hline & $\begin{array}{l}\text { 1.8. La jornada en que se asiste a clases afecta significativamente la } \\
\text { deserción de los estudiantes. }\end{array}$ \\
\hline \multirow{4}{*}{$\begin{array}{l}\text { 2. ¿Qué variables explican } \\
\text { significativamente la deserción } \\
\text { de los estudiantes de educación } \\
\text { superior en cada una de las } \\
\text { escuelas? }\end{array}$} & $\begin{array}{l}\text { 2.1. El promedio de las notas de enseñanza media afecta de manera } \\
\text { significativa la deserción de los estudiantes de la escuela de } \\
\text { (administración y negocios o comunicación o diseño o informática y } \\
\text { telecomunicaciones o salud). }\end{array}$ \\
\hline & $\begin{array}{l}\text { 2.2. La evaluación diagnóstica de lenguaje afecta significativamente la } \\
\text { deserción de los estudiantes de la escuela de (administración y negocios } \\
\text { o comunicación o diseño o informática y telecomunicaciones o salud). }\end{array}$ \\
\hline & $\begin{array}{l}\text { 2.3. La evaluación diagnóstica de matemática afecta significativamente } \\
\text { la deserción de los estudiantes de la escuela de (administración y } \\
\text { negocios o comunicación o diseño o informática y telecomunicaciones } \\
\text { o salud). }\end{array}$ \\
\hline & $\begin{array}{l}\text { 2.4. El establecimiento educacional de procedencia afecta de } \\
\text { manera significativa la deserción de los estudiantes de la escuela de } \\
\text { (administración y negocios o comunicación o diseño o informática y } \\
\text { telecomunicaciones o salud). }\end{array}$ \\
\hline
\end{tabular}




\begin{tabular}{ll}
\hline \multicolumn{1}{c}{ Preguntas } & \multicolumn{1}{c}{ Hipótesis de trabajo (o investigación) } \\
\hline & $\begin{array}{l}\text { 2.5. El año de egreso de la enseñanza media afecta de manera } \\
\text { significativa la deserción de los estudiantes de la escuela de } \\
\text { (administración y negocios o comunicación o diseño o informática y } \\
\text { telecomunicaciones o salud). }\end{array}$ \\
\hline $\begin{array}{l}\text { 2.6. El género del alumno afecta significativamente la deserción del } \\
\text { estudiante de la escuela de (administración y negocios o comunicación } \\
\text { o diseño o informática y telecomunicaciones o salud). }\end{array}$ \\
\hline $\begin{array}{l}\text { 2.7. El lugar de procedencia del alumno afecta de manera significativa la } \\
\text { deserción del estudiante de la escuela de (administración y negocios o } \\
\text { comunicación o diseño o informática y telecomunicaciones o salud). }\end{array}$ \\
\hline $\begin{array}{l}\text { 2.8. La jornada en que se asiste a clases afecta significativamente la } \\
\text { deserción de los estudiantes de la escuela de (administración y negocios } \\
\text { o comunicación o diseño o informática y telecomunicaciones o salud). }\end{array}$ \\
\hline $\begin{array}{l}\text { 3. ¿Cómo se organizan las } \\
\text { dinámicas de deserción de los } \\
\text { estudiantes de la educación } \\
\text { superior en cada una de las } \\
\text { escuelas? }\end{array}$ & \begin{tabular}{l} 
la educación superior es diferenciada para cada una de las escuelas. \\
\hline
\end{tabular} \\
\hline
\end{tabular}

\section{Técnica de recolección y análisis de datos}

El proceso de recolección de los datos considera la consolidación y la sistematización de información en torno a las variables definidas, de acuerdo a los reportes obtenidos del sistema de seguimiento de los estudiantes con el que cuenta la institución Duoc Uc. En este sentido, los reportes obtenidos provienen de una base de datos con la que cuenta la organización educativa en la que se encuentra el registro del tipo de establecimiento de egreso de los alumnos, la nota de enseñanza media con la que se finalizó el nivel educacional secundario, los resultados obtenidos en le evaluación diagnóstica tanto de lenguaje como matemática. Además, a través de este mismo sistema de seguimiento, existe información referente a los estudiantes desertores, la comuna de procedencia, el género, el año de egreso de la enseñanza media, la jornada en que asiste a las clases.

Las variables seleccionadas responden a lo señalado en el marco conceptual en torno a la deserción estudiantil en el contexto de la educación supe- 
rior y particularmente lo referido a los distintos modelos asociados al estudio de este fenómeno. Al respecto, a través de éstos se precisan qué factores tanto individuales como organizacionales influyen en el proceso de abandono, pero para efectos de esta investigación únicamente hemos trabajado con aquellas variables individuales a las que podemos acceder desde el primer día de clases de los estudiantes en la institución. En este sentido, cada una de ellas se relacionan con aspectos de los ámbitos académico, demográfico y organizacional, tales como: las habilidades y/o conocimientos de entrada en áreas de formación transversal, el género, el lugar de residencia, el promedio de enseñanza media, el año de egreso de la enseñanza media, el tipo de establecimiento, la jornada en que asiste a clases.

Cada uno de los datos que fueron presentados de manera previa se analizaron con el apoyo del software de análisis estadístico R. En concordancia con los objetivos de la investigación y las características de las variables que fueron consideradas para definir qué factores permiten explicar de mejor forma la deserción de los estudiantes en una institución de educación superior técnico-profesional, se implementó un modelo lineal generalizado con distribución de errores binomiales y la función de vínculo Logit, viéndose reflejado en la siguiente fórmula.

Figura 1

Fórmula Modelo Lineal Generalizado con distribución Binomial y factor de vínculo Logit

$y_{i}=\sum_{j} \beta_{j} X_{i j}+\varepsilon_{i}$
siendo,
$\mu_{i}=E\left(Y_{i}\right):$ valores ajustados variable respuesta
$\eta_{i}=\sum_{j} \beta_{j} X_{i j}:$ predictores
$\varepsilon_{i}:$ componente aleatorio
$\eta_{i}=g\left(\mu_{i}\right):$ función de enlace cambia cuando los componentes aleatorios no siguen
una distribución normal




\section{Resultados}

A partir del análisis realizado a través del software y de la implementación del modelo lineal generalizado con distribución de errores binomial y un factor de vínculo logit, fue posible reconocer aquellas variables de la muestra que tenían una mayor incidencia en el fenómeno de la deserción. Este procedimiento también se implementó por cada escuela y estos hallazgos han posibilitado el establecimiento de diferencias entre las mismas.

Tabla 11

Análisis general de las variables seleccionadas que han sido consideradas como factores que influyen en el proceso de deserción

\begin{tabular}{lcccc}
\hline & Estimate & Std. Error & z value & $\operatorname{Pr}(>|\mathrm{z}|)$ \\
\hline Intercept) & -2.216418 & 1.706749 & -1.299 & 0.194074 \\
\hline DLENG & -0.007456 & 0.006524 & -1.143 & 0.253068 \\
\hline DMAT & 0.005989 & 0.005708 & 1.049 & 0.294076 \\
\hline EGRESO_EM & $-\mathbf{0 . 1 1 5 0 1 4}$ & $\mathbf{0 . 0 2 9 6 2 5}$ & $\mathbf{- 3 . 8 8 2}$ & $\mathbf{0 . 0 0 0 1 0 3 * * *}$ \\
\hline ESTABLECIMIENTO & -0.114121 & 0.114955 & -0.993 & 0.320835 \\
\hline PROM_EM & 0.288283 & 0.272756 & 1.057 & 0.290544 \\
\hline SEXO & -0.518907 & $\mathbf{0 . 2 2 4 5 5 2}$ & $\mathbf{- 2 . 3 1 1}$ & $\mathbf{0 . 0 2 0 8 4 1 *}$ \\
\hline JORNADA & $\mathbf{0 . 5 1 9 8 9 6}$ & $\mathbf{0 . 3 0 5 8 6 8}$ & $\mathbf{1 . 7 0 0}$ & $\mathbf{0 . 0 8 9 1 8 0}$ \\
\hline COMUNA & -0.005477 & 0.004356 & -1.257 & 0.208663 \\
\hline
\end{tabular}

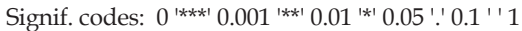

De acuerdo con el resultado a partir del análisis de los datos, son tres las variables que tienen impacto en el proceso de deserción a nivel institucional y, tal como se visualiza en la tabla 10, cada una de ellas se corresponde con una de las tres dimensiones en la que se enmarca este trabajo. En este contexto, la jornada a la que se asiste a clases, ya sea diurna o vespertina, se transforma en uno de los factores que podría influir en la opción de dejar de lado el pro- 
yecto de formarse profesionalmente. Esto se corrobora observando que dicha variable logra un p-valor del 0,08, por tanto, debe ser considerado como un factor que permitiría predecir el abandono de la institución educativa con un nivel de confianza del $90 \%$.

En relación con lo señalado, los procesos de toma de decisiones deberán orientarse a entregar un mejor servicio a los estudiantes y sobre todo de la jornada vespertina. Esto, mediante procesos de acompañamiento como talleres, instancias de seguimiento, diálogo y otros, a fin de lograr obtener información de cuál es la situación por parte de los estudiantes, así como también, buscando que valoren la institución en la que se encuentran y perciban más beneficios que costos. En conexión con la teoría, las acciones de este tipo cobran relevancia dado que cada estudiante siempre estará evaluando cuáles son los beneficios que está teniendo, y en la medida que logre visualizar que estos superan los costos, permanecerá en la institución.

Otro de los aspectos a considerar está en relación con el género de los desertores. En este sentido, la mayor parte son mujeres, cuyo porcentaje respecto del total de alumnos desertores (98) equivale al 57\%, mientras que el $43 \%$ corresponde a hombres. Es importante precisar que del total de mujeres que ha optado por abandonar, el 40\% de ellas son estudiantes de la escuela de administración y negocios, lo que nos podría llevar a inferir que hay factores particulares de su experiencia en esa unidad que están influyendo al momento de tomar la decisión de continuar o abandonar.

La tercera variable corresponde al año de egreso de la enseñanza media, tal como se aprecia en la tabla 10 es el factor con mayor significancia estadística. En este caso se hace necesario precisar que se da un fenómeno particular, pues la mayor cantidad de estudiantes que ha desertado en el rango de años estudiados corresponden a alumnos que egresaron entre los años 2012-2015, mientras que los estudiantes que menos desertan corresponden a aquellos que egresaron de la educación secundaria en periodos anteriores al año 2000. En conexión con el modelo psicológico, esto tendría relación con el nivel de madurez de los individuos y la claridad en sus metas, situación que en alguna medida vendría a explicar el que la mayor parte de los alumnos que han egresado en periodos previo al 2000 de la enseñanza se mantengan en la institución, mientras que aquellos pertenecientes, sobre todo, a años de egreso entre 2012 a 2015 tiendan a abandonar. Esto significaría por tanto 
que aquellos que renuncian no tienen claridad de si el área en la que se están formando o incluso el lugar es lo que realmente desean, produciéndose de este modo un debilitamiento de expectativas iniciales.

La situación descrita no es homogénea para todas las escuelas, pues Comunicación, Diseño y Salud, no reportaron resultados que dieran cuenta de variables que afectaran significativamente a la deserción. En tanto, las escuelas de Administración y Negocios e Informática y Telecomunicaciones presentaron dinámicas de comportamiento distintas.

Tabla 12

Análisis de variables que influyen en el proceso de deserción en la escuela de Informática y Telecomunicaciones

\begin{tabular}{lcccc}
\hline & Estimate & Std. Error & z value & $\operatorname{Pr}(>|\mathbf{z}|)$ \\
\hline (Intercept) & -7.490315 & 8.635845 & -0.867 & 0.385749 \\
\hline DLENG & -0.015243 & 0.016741 & -0.911 & 0.362555 \\
\hline DMAT & -0.005440 & 0.013545 & -0.402 & 0.687945 \\
\hline CODIGO_ANO_EGRESO & -0.055587 & 0.064931 & -0.856 & 0.391947 \\
\hline CODIGO_COMUNA & -0.004896 & 0.012397 & -0.395 & 0.692882 \\
\hline CODIGO_ESTABLECIMIENTO & 0.278548 & 0.981 & 0.326384 & 0.283820 \\
\hline CODIGO_GENERO & -2.046816 & 0.586702 & -3.489 & $0.000485 * * *$ \\
\hline CODIGO_JORNADA & -0.339439 & 0.580935 & -0.584 & 0.559019 \\
\hline PROM.EM & -0.143533 & 0.677712 & -0.212 & 0.832270 \\
\hline
\end{tabular}

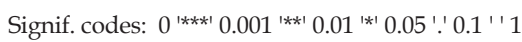

Tal como se aprecia, la única variable que estaría aportando a la explicación de abandono en esta escuela está en relación con el género, siendo el 100\% de las deserciones del género femenino. Al realizar una conexión de esta información con los modelos psicológicos y sociológicos, específicamente en su componente de integración social, esta situación responde a que las ca- 
rreras que conforman la escuela de informática se destacan por tener un alto porcentaje de varones, en muchos casos existen cursos completos que únicamente tienen hombres. La presencia del género femenino en esta escuela es menor, tanto a nivel de docentes como de estudiantes, lo que es posible corroborar al ingresar a una sala de clases de las carreras del área. Dada esta situación, es preciso tomar decisiones al respecto, a nivel organizacional, en torno a la dimensión de integración social para poder responder a las expectativas de las alumnas $\mathrm{y}$, a su vez, mejorar las tasas de permanencia del género femenino.

En la escuela de Administración es posible visualizar que la dinámica de deserción no es igual, pero se comparte un aspecto referido a la variable de género como factor explicativo del fenómeno. No obstante, se agrega una segunda variable, la que dice relación con el tipo de establecimiento del cual egresó el estudiante. En la tabla 12 es posible visualizar en qué medida cada una de las variables logra impactar de manera significativa en la deserción.

Tabla 13

Análisis de las variables que influyen en el proceso de deserción en la escuela de Administración y Negocios

\begin{tabular}{lcccc}
\hline & Estimate & Std. Error & z value & $\operatorname{Pr}(>|\mathrm{z}|)$ \\
\hline (Intercept) & -2.350345 & 2.274500 & -1.033 & 0.3014 \\
\hline DLENG & -0.004884 & 0.009074 & -0.538 & 0.5904 \\
\hline DMAT & 0.011731 & 0.008186 & 1.433 & 0.1518 \\
\hline CODIGO_ANO_EGRESO & -0.007351 & 0.031813 & -0.231 & 0.8173 \\
\hline CODIGO_CARRERA & -0.014691 & 0.028424 & -0.517 & 0.6053 \\
\hline CÓDIGO_COMUNA & -0.005659 & 0.005932 & -0.954 & 0.3401 \\
\hline CODIGO_ESTABLECIMIENTO & -0.330124 & $\mathbf{0 . 1 6 3 4 4 5}$ & $\mathbf{- 2 . 0 2 0}$ & $\mathbf{0 . 0 4 3 4}$ \\
\hline CODIGO_GENERO & $-\mathbf{0 . 5 4 3 4 7 5}$ & $\mathbf{0 . 3 2 5 1 3 7}$ & $\mathbf{- 1 . 6 7 2}$ & $\mathbf{0 . 0 9 4 6}$ \\
\hline CODIGO_JORNADA & 0.143527 & 0.300753 & 0.477 & 0.6332 \\
\hline
\end{tabular}




\begin{tabular}{|c|c|c|c|c|}
\hline & Estimate & Std. Error & $\mathrm{z}$ value & $\operatorname{Pr}(>|z|)$ \\
\hline PROM.EM & 0.233057 & 0.352340 & 0.661 & 0.5083 \\
\hline
\end{tabular}

Tal como se observa en los datos, el género también juega un rol clave en esta escuela, pues del total de estudiantes que desertaron de la escuela, el 71\% corresponde al género femenino, mientras que el $29 \%$ al masculino. Esto podría conectarse con factores más específicos asociados al contexto familiar o laboral, dado que del total de mujeres que desertaron, el 58,9\% se encontraba matriculada en jornada vespertina, ya sea presencial o semipresencial.

Así también, es importante considerar el tipo de establecimiento de egreso de secundaria, pues las características específicas de la institución permitirían explicar quienes tienen una mayor probabilidad de abandonar. En este caso, del total de desertores de la escuela, el 51\% provenía de establecimientos municipales técnico-profesional, seguido de un $29 \%$ por parte de estudiantes de liceos particulares subvencionados científico-humanistas, un $11 \%$ de alumnos de establecimientos particulares subvencionados técnicoprofesionales y un $9 \%$ de colegios municipales científico-humanistas. Dado esto, se hace necesario incorporar acciones tanto desde la dimensión social como de la dimensión académica, a nivel organizacional, que permitan entregar apoyo y fortalecer algunas competencias de entrada necesarias, de tal manera que los estudiantes puedan contar con más herramientas para enfrentar la educación superior.

Para efectos de síntesis, a continuación, se presenta un resumen con las hipótesis que se lograron aceptar. 
Tabla 14

Síntesis de comprobación de hipótesis de investigación

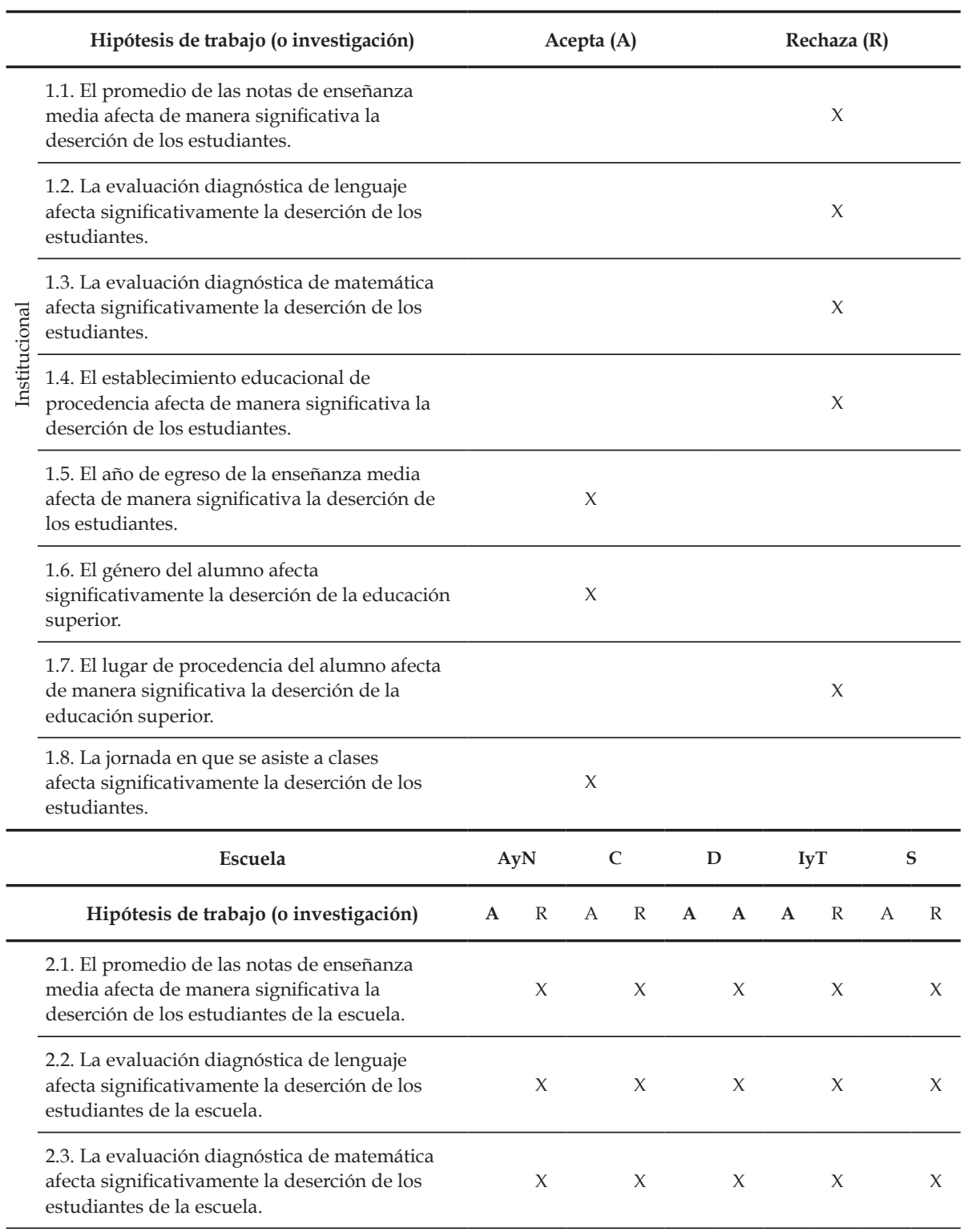




\begin{tabular}{|c|c|c|c|c|c|c|c|c|c|c|}
\hline Escuela & \multicolumn{2}{|c|}{ AyN } & \multicolumn{2}{|c|}{$\mathrm{C}$} & \multicolumn{2}{|c|}{ D } & \multicolumn{2}{|c|}{ IyT } & \multicolumn{2}{|c|}{ s } \\
\hline Hipótesis de trabajo (o investigación) & $\mathbf{A}$ & $\mathrm{R}$ & A & $\mathrm{R}$ & A & A & A & $\mathrm{R}$ & A & $\mathrm{R}$ \\
\hline $\begin{array}{l}\text { 2.4. El establecimiento educacional de } \\
\text { procedencia afecta de manera significativa la } \\
\text { deserción de los estudiantes de la escuela. }\end{array}$ & $x$ & & & $x$ & & $x$ & & $x$ & & $x$ \\
\hline $\begin{array}{l}\text { 2.5. El año de egreso de la enseñanza media } \\
\text { afecta de manera significativa la deserción de } \\
\text { los estudiantes de la escuela. }\end{array}$ & & $x$ & & $x$ & & $x$ & & $x$ & & $\mathrm{x}$ \\
\hline $\begin{array}{l}\text { 2.6. El género del alumno afecta } \\
\text { significativamente la deserción del estudiante } \\
\text { de la escuela. }\end{array}$ & $x$ & & & $x$ & & $x$ & $x$ & & & $\mathrm{x}$ \\
\hline $\begin{array}{l}\text { 2.7. El lugar de procedencia del alumno } \\
\text { afecta de manera significativa la deserción del } \\
\text { estudiante de la escuela. }\end{array}$ & & $x$ & & $x$ & & $x$ & & $x$ & & $x$ \\
\hline $\begin{array}{l}\text { 2.8. La jornada en que se asiste a clases } \\
\text { afecta significativamente la deserción de los } \\
\text { estudiantes de la escuela. }\end{array}$ & & $x$ & & $x$ & & $x$ & & $x$ & & $x$ \\
\hline Hipótesis de trabajo (o investigación) & \multicolumn{4}{|c|}{ Acepta (A) } & & & \multicolumn{4}{|c|}{ Rechaza (R) } \\
\hline $\begin{array}{l}\text { 3.1. La organización de las dinámicas de } \\
\text { deserción de los estudiantes de la educación } \\
\text { superior es diferenciada para cada una de las } \\
\text { escuelas. }\end{array}$ & & & $x$ & & & & & & & \\
\hline
\end{tabular}

\section{Conclusiones}

Con todos los datos expuestos y análisis realizados, se hace necesario, por una parte, indagar aún más en otros aspectos a fin de poder lograr a un modelo más nutrido y que integre una mayor diversidad de variables, de acuerdo con lo definido por la literatura en torno a los factores que podrían predecir o el abandono de la educación superior. Del mismo modo, para poder nutrir y contar con un mejor modelo, será requisito contar con información más detallada respecto de los estudiantes a fin de intentar que el modelo que se proponga sea efectivamente multicausal. 
Por otra parte, es importante considerar que, a raíz de los resultados obtenidos, la organización estudiada deberá implementar algunas acciones orientadas a disminuir los índices de deserción tanto a nivel general como para cada escuela. Para estos efectos, será necesario contar con programas de acompañamiento y/o apoyo a los estudiantes en distintas áreas, vale decir, que vayan desde lo netamente disciplinar a aspectos más sociales, permitiendo de esta manera que al momento de que cada alumno realice el proceso de evaluación de los costos y beneficios que está teniendo, logren ser mayores los beneficios y, por ende, exista una motivación a permanecer en la casa de estudios.

Finalmente, tal como se ha mencionado, sería importante poder nutrir mucho más este estudio integrando especificaciones en torno a la decisión de desertar, a fin de clasificarla, por ejemplo, de acuerdo con la tipología propuesta realizada por Páramo y Correa (1999) e identificar cuál es el tipo de deserción que más se da en la organización educativa y, a su vez, cuáles son las variables que explicarían el fenómeno.

\section{Referencias}

Aparicio, Miriam (2008). Las causas de la deserción en las Universidades Nacionales. San Juan-Argentina: Editorial FFHA-UNSJ.

Aparicio, Miriam T. (2012). La deserción universitaria como parámetro de calidad y su relación con factores psicosociales. Diálogo, (20), p-145.

Apaza, Effer, y Huamán, Francisco (2012). Factores determinantes que inciden en la deserción de los estudiantes universitarios-Determinants that affect the defection of college students. Apuntes Universitarios, (1).

Ariza, Sara, y Arias, Daniel (2014). Factores intervinientes en la deserción escolar de la Facultad de Psicología, Fundación Universitaria Los Libertadores. Tesis Psicológica, 4 (1), 72-85.

Barrios, M. Andrés (2011). Deserción y financiamiento en las universidades chilenas. (Tesis de maestría no publicada. Santiago, Pontificia Universidad Católica de Chile, Chile.

Bean, John P. (1980). Dropouts and turnover: The synthesis and test of a causal model of student attrition. Research in higher education, 12 (2), 155-187. 
Berger, Joseph B., y Milem, Jeffrey F. (2000). Exploring the impact of historically Black colleges in promoting the development of undergraduates' self-concept. Journal of College Student Development, 41 (4).

Braxton, John M.; Shaw Sullivan, Ana V., y Johnson, Robert M. (1997). Appraising Tinto's theory of college student departure. Higher education-new york-agathon press incorporated, 12, 107-164.

Castaño, Elkin; Gallón, Santiago; Gómez, Karoll, y Vásquez J.(2004) Deserción estudiantil universitaria: una aplicación de modelos de duración. Lecturas de Economía (60), 30-65.

Celis, Rubén; Flores, Carmen; Reyes, María, y Venegas, Héctor (2013). Factores de riesgo de deserción presentes en alumnos repitentes de las carreras de enfermería y kinesiología en una universidad chilena. Ciencia y enfermería, 19 (3), 63-71.

Colmenares, Mercedes, y Delgado, Flor (2008). La correlación entre rendimiento académico y motivación de logro: elementos para la discusión y reflexión. REDHECS: Revista electrónica de Humanidades, Educación y Comunicación Social, 3 (5), 179-191.

De los Ríos, Danae, y Canales, Andrea (2007). Factores explicativos de la deserción universitaria. Calidad en la Educación, (26), 173-201.

Díaz, Christian (2008). Modelo conceptual para la deserción estudiantil universitaria chilena. Estudios pedagógicos (Valdivia), 34 (2), 65-86.

Donoso, Sebastián, y Schiefelbein, Ernesto (2007). Análisis de los modelos explicativos de retención de estudiantes en la universidad: una visión desde la desigualdad social. Estudios pedagógicos (Valdivia), 33 (1), 7-27.

García, Ana (2014). Rendimiento académico y abandono universitario: Modelos, resultados y alcances de la producción académica en la Argentina. Revista Argentina de Educación Superior, (8), 9-38.

González, Luis, y Uribe, Daniel (2005). Estimaciones sobre la repitencia y deserción en la educación superior. Revista Calidad de la Educación, (17), 91-108.

González, Luis E. (2006). Repitencia y deserción en América Latina. Disponible en: http:/ / www. intercontacto.com/gente.

Gordillo, Esther, y Polanco, Jorge (1970). Deserción estudiantil: análisis cuantitativo. Oficina de Planeación, División de Programación Económica, Universidad Nacional de Colombia, Bogotá, DC.

Himmel, Erika (2002). Modelos de análisis de la deserción estudiantil en la educación superior. Revista Calidad en la educación, 17 (2), 91-108.

Huesca, Gabriela, y Castaño, Belén (2007). Causas de deserción de alumnos de primeros semestres de una universidad privada. Revista Mexicana de Orientación Educativa, 5 (12), 34-39. 
Larroucau, Tomás (2013). Estudio de los factores determinantes de la deserción, en el sistema universitario chileno.

Malagón, L.; Soto, L., y Eslava, P. (2007). La deserción en la Universidad de los Llanos (1998-2004). Orinoquia, 11 (1), 23-40.

Medellín, Elvers (2010). Contrastación de dos modelos motivacionales de autodeterminación para predecir la deserción en universitarios. Acta Colombiana de Psicología, 13 (2), 57-68.

Microdatos, C. U. (2008). Estudio sobre causas de la deserción universitaria. Santiago de Chile.

Mizala, A.; Hernández, T., \& Makovec, M. (2011). Fonide 511059: Determinantes de la elección y deserción en la carrera de Pedagogía. Santiago.

Mori, María (2014). Deserción universitaria en estudiantes de una universidad privada de Iquitos. Revista Digital de Investigación en Docencia Universitaria (RIDU).

Olave, G., Rojas, I., \& Cisneros, M. (2013). Deserción universitaria y alfabetización académica. Educación y educadores, 16 (3), 455-471.

Páramo, Gabriel y Correa, Carlos (1999). Deserción estudiantil universitaria. Revista universitaria. Cuaderno de Humanidades, (8).

Rodríguez, Javier y Hernández, Juan (2008). La deserción escolar universitaria en México. La experiencia de la Universidad Autónoma Metropolitana, Campus Iztapalapa/The university scholastic desertion in Mexico. The experience of the Universidad Autónoma Metropolitana Campus Iztapalapa. Actualidades Investigativas en educación, 8 (1).

Román, Carola (2013). Más programas propedéuticos en Chile: El discurso de los estudiantes en la Universidad Católica Silva Henríquez. Estudios pedagógicos (Valdivia), 39 (2), 263-278.

Soria, Karla, y Zúñiga, Sergio (2014). Aspectos Determinantes del Éxito Académico de Estudiantes Universitarios. Formación universitaria, 7 (5), 41-50.

Spady, William G. (1970). Dropouts from higher education: An interdisciplinary review and synthesis. Interchange, 1 (1), 64-85.

St. John, E. P., Cabrera, A. F., Nora, A., y Asker, E. H. (2000). Economic influences on persistence reconsidered: How can finance research inform the reconceptualization of persistence models. Reworking the student departure puzzle, 1, 29-47.

Tinto, Vincent (1975). Drop out from Higher Education: A Theoretical Synthesis of Recent Research. Review of Educational Research, 45 (1), 89-195.

Tinto, Vincent (1982). Definir la Deserción: Una Cuestión de Perspectiva. Revista de Educación Superior, 71, 33-51. 
Torres, Luz (2012). Retención estudiantil en la educación superior: revisión de la literatura y elementos de un modelo para el contexto colombiano. Bogotá: Editorial Pontificia Universidad Javeriana.

Villa, M., y Barriga, Omar (2010). Adaptación del modelo de deserción universitaria de Tinto a la Universidad Católica de la Santísima Concepción, Chile. Revista de Ciencias Sociales, 16 (4).

Vries, Wietse de; León, Patricia; Romero, José y Hernández, Ignacio (2011). ¿Desertores o decepcionados? Distintas causas para abandonar los estudios universitarios. Revista de la educación superior, 40 (160), 29-49.

Viale, Héctor (2014). Una aproximación teórica a la deserción estudiantil universitaria. Revista digital de Investigación en Docencia universitaria, 8 (1), 59-76. 
